\title{
APRENDIZAJE BASADO EN PROBLEMAS: UNA HERRAMIENTA ACADÉMICA DE AUTO APRENDIZAJE EN LOS ESTUDIANTES DE SEXTO NIVEL DE LA CARRERA DE PSICOLOGÍA QUE CURSAN LA MATERIA DE TRASTORNOS DE PERSONALIDAD
}

LEARNING BASED ON PROBLEMS: AN ACADEMIC TOOL OF SELF-LEARNING IN SIXTH GRADE STUDENTS OF THE CARRER OF PSYCHOLOGY WHO TAKE THE PERSONALITY DISORDERS SUBJECT

ALEJANDRA DÍAZ T. ${ }^{i}$

PAULA LÁrRAGA O.

BELÉN MIÑO T. JESSY ROBAYO CH.*

Recibido: 24 de julio de 2019 Aceptado: 1 de noviembre de 2019

'Universidad Politécnica Salesiana, Facultad de Psicología, Quito, Ecuador. 



\section{APRENDIZAJE BASADO EN PROBLEMAS: UNA HERRAMIENTA ACADÉMICA DE AUTO APRENDIZAJE EN LOS ESTUDIANTES DE SEXTO NIVEL DE LA CARRERA DE PSICOLOGÍA QUE CURSAN LA MATERIA DE TRASTORNOS DE PERSONALIDAD}

LEARNING BASED ON PROBLEMS: AN ACADEMIC TOOL OF SELF-LEARNING IN SIXTH GRADE STUDENTS OF THE CARRER OF PSYCHOLOGY WHO TAKE THE PERSONALITY DISORDERS SUBJECT

Alejandra Díaz T., Paula Lárraga O., Belén Miño T., Jessy Robayo CH.

Palabras clave: ABP, método de aprendizaje, estrategias de autoaprendizaje, $A B P$ como herramienta $A B P$ en la psicología Keywords: ABP, learning method, self-learning strategies, ABP as a tool, $\mathrm{ABP}$ in psychology 


\section{RESUMEN}

El ABP (aprendizaje basado en problemas), es un método de aprendizaje moderno que varios docentes han optado por aplicar a sus estudiantes, dejando de lado el método tradicional de enseñanza. El foco o eje principal de este método son los estudiantes, especialmente los que pertenecen a estudios superiores, los cuales adquieren por su propia cuenta con la supervisión y ayuda de su tutor a cargo: conocimientos, habilidades y aptitudes a través de situaciones de la vida real asociadas a la teoría revisada en clase.

El objetivo de este trabajo es conocer la efectividad del ABP como herramienta académica en los estudiantes de sexto nivel de la carrera de Psicología que cursan la materia de trastornos de personalidad.
Para evidenciar el correcto uso y el aprendizaje obtenido por los estudiantes de sexto nivel (grupo uno y dos) de psicología laboral, se utilizará el método cuantitativo a través de encuestas online, con el fin de analizar y comprobar información y datos obtenidos para lograr el objetivo planteado.

Como conclusión hemos logrado conocer el proceso y su impacto con oportunidades u obstáculos causados por el método ABP en los estudiantes, con el fin de evidenciar capacidades y aptitudes adquiridas por los equipos de trabajo a lo largo del semestre, garantizando la efectividad de este nuevo método de estudio.

\section{ABSTRACT}

ABP (Problem-based learning) is a modern learning method that several teachers have elected to use in their classrooms, leaving aside the traditional teaching methods. The focal point of this method are the students, especially those in higher education, who, on their own, with the supervision and assistance of their instructor, acquire knowledge, skills, and capabilities in handling re- al-world experiences through practical theory.

The objective is to know how effective $A B P$ is as an academic learning method for sixth-level Psychology students studying personality disorders.

In order to demonstrate the proper use of $A B P$ and the learning gained by sixth-level occupational psychology students (groups one and two), the quantitative 
method will be used by means of online surveys, with the purpose of analyzing and verifying information and data obtained to achieve the objective set.

Finally, we get to know the process and its impact by the opportunities or the obstacles caused by the ABP method on students in order to demonstrate the skills and aptitudes acquired by the groupings throughout the semester of this method of study's implementation.

\section{INTRODUCCIÓN}

El ABP es considerado como un proceso de enseñanza constructivista, ya que es un método que permite al estudiante asumir los roles de un docente, es por eso que se considera una de las mejores técnicas de enseñanza, ya que promueve una metodología distinta a la convencional.

Según Fernandez (2016), este método educativo se enfoca en el aprendizaje, en la reflexión, y en la indagación, por parte de los alumnos; es ahí que el profesor pasa a actuar como guía o facilitador del proceso para determinar posibles soluciones al tema; adicionalmente, el autor toma en cuenta todo el procedi- miento que se da dentro del aprendizaje desde la creación de un proyecto hasta la continuidad que este tiene en base a la búsqueda de información y finalmente a la resolución del problema.

Es por eso que se considera el uso de esta técnica como fundamental, puesto que permite adaptarse a diversos ámbitos ya sea administrativos, pedagógicos e institucionales; tomando en cuenta que se utiliza con la finalidad de amplificar el nivel de control, modificar el diseño de los procesos a investigar, y a su vez extiende sus habilidades de razonamiento y habilidades de pensamiento crítico.

\section{MARCO TEÓRICO}

\section{ABP (aprendizaje basado en problemas)}

El aprendizaje basado en problemas (ABP), es una estrategia educacional que permite al estudiante adquirir conocimientos, habilidades y actitudes, a través de una situación de la vida real; a su vez, favorece el pensamiento crítico para la resolución de problemas tanto académicos como laborales. Una de las 
características principales que ofrece el $\mathrm{ABP}$ es que concibe al estudiante como protagonista en la gestión de su propio aprendizaje, ya que ejerce el control de la situación que se le presente, tomando en cuenta que el ABP le permitirá elegir su ritmo para la resolución del problema.

En el análisis y descripción de Carretero (1993), se resalta que los principios del ABP se conciben dentro de la percepción constructivista, puesto que permite a la persona construir un aprendizaje mediante la experimentación y reorganización de ideas internas, con el fin de que la persona pueda comprender y asimilar la información recibida. Dando como resultado la ampliación y adquisición de nuevos conocimientos.

\section{Roles dentro del $A B P$}

De acuerdo a la metodología del ABP, cada miembro desempeña un rol diferente, puesto que es un metodo que favorece al aprendizaje de los estudiantes mediante el trabajo autónomo, es por eso que se ofrecen los siguientes roles, según Carretero (1993):

- Rol del docente; su rol es de ser el tutor, el cual facilita y fomenta a los estudiantes a ciertas actividades de reflexión y búsqueda, con el fin de que puedan identificar sus propias necesidades de aprendizaje.
Para ello existen varias características que requiere tener un docente en el aprendizaje basado en problemas, dentro de ellas se encuentra una actitud positiva, estar formado y capacitado para aplicar el método ya que el tutor actúa como guía mediante el proceso. Sin embargo, en el ABP no solo aprende el estudiante sino también el docente ya que se encuentra en continuo aprendizaje.

- Rol del estudiante; el papel del estudiante es de participación activa dentro del proceso ABP, pues actúa como protagonista del mismo ya que todo el proceso es por y para su aprendizaje.

\section{Funcionamiento del ABP}

Según Morales (2018), dentro de la estructura organizacional del ABP, se nombra entre los estudiantes un coordinador, el cual es el encargado de asignar a cada uno de los miembros un rol participativo en la toma de decisiones referente a la búsqueda de posibles soluciones para la problemática propuesta. Por otra parte, el docente permanece como recurso dentro de la actividad colectiva, con un perfil bajo, interviniendo solo si el grupo se desvía de su objetivo. Hoy en día puede desarrollarse hasta con 60 estudiantes, destacando en cada grupo un tutor con el fin de que la metodología sea llevada de la mejor manera. 


\section{ABP como método de aprendizaje}

El ABP es considerado un método didáctico, que cae en el dominio de la pedagogía activa, ya que dicho método procede de manera sistemática y a su vez motiva a los estudiantes a un aprendizaje independiente, generando nuevas alternativas propias para la resolución de problemas. Para Bruner, citado por (Restrepo, 2005), es fundamental llevar al aprendizaje humano más allá de la mera información, es decir se requiere llevarla hacia los objetivos de aprender, comprender y resolver problemas; con este fin, propone siete métodos que pueden operacionalizar el aprendizaje, dentro de los cuales se encuentran:

- El seminario investigativo.

- El ABP en sus distintas modalidades.

- El método de proyectos.

- El método tutorial.

- El estudio de casos.

- La enseñanza personalizada.

- Simulación y juegos.

\section{ABP estrategia de autoaprendizaje}

Se considera al aprendizaje basado en problemas como una estrategia de autoaprendizaje, ya que permite al estudiante ser el protagonista esencial de su aprendizaje, pues lo reta a buscar distintas alternativas de respuestas ante una problemática, esto permite que se desarrolle su autonomía, fomente su espíritu crítico y así se promueva la creatividad.

Según Restrepo (2005), el estudiante aprende por cuenta propia a construir conocimientos empíricos sobre una problemática propuesta, es así como esta estrategia permite también la interacción con otros dando como resultado un aprendizaje estructurado que pueda responder las necesidades dentro de la realidad.

\section{ABP como herramienta académica}

La obtención de información nueva, convierte al ABP en una de las herramientas fundamentales para el trabajo autónomo, puesto que se la considera como una herramienta activa para el docente, la cual posee la finalidad de promover la participación activa de los estudiantes, permitiéndoles investigar, indagar, dando como resultado el autoaprendizaje. Es por eso que para Chavez, Gonzalez, \& Hidalgo (2016), el ABP también es considerado como:

- Un gestionador de conocimientos.

- Una práctica reflexiva que permita reflexionar sobre problemas singulares, inciertos y complejos.

Una fuente para la adaptación de cambios.

En este artículo también se toma- 
rá en cuenta la perspectiva psicológica dentro del aprendizaje basado en problemas (ABP).

\section{ABP en la psicología}

El aprendizaje basado en problemas surge como reacción de la enseñanza tradicional; sin embargo, su uso y aplicación se extiende en campos educativos y sirve para la formación universitaria de profesionales. Dentro de la psicología se define al ABP como un modelo de enseñanza/aprendizaje, en el cual se combinan conocimientos, habilidades y actitudes, los mismos que son útiles para la práctica profesional a través del trabajo en equipo con el fin de solucionar los problemas propios de una profesión.
Según Restrepo (2005), se proyecta al ABP dentro de la asignatura de Psicopedagogía; esto, con la finalidad de examinar y evaluar este método en el entorno educativo universitario, ya que su metodología se enfoca principalmente en que el estudiante adquiera conocimientos, habilidades y actitudes a través de una situación de la vida real.

Para esto se propone cuatro momentos fundamentales dentro de la Psicopedagogía que permiten que el método ABP se elabore de una manera correcta:

- Momento l: Fase de preparación.

- Momento Il: Fase de planificación.

- Momento III: Fase de Acción.

- Momento IV: Fase de Evaluación.

\section{METODOLOGÍA /MATERIALES}

Para la recolección de datos que nos permitirán lograr el objetivo planteado, se ha previsto utilizar el método cuantitativo, el cual según Sanz (2017), es un procedimiento que se basa en la utilización de los números para analizar, investigar y comprobar tanto información como datos. La investigación o metodología cuantitativa se produce por la causa y efecto de las cosas, y es uno de los métodos más conocidos y utilizados.
Es así que se optó por la aplicación de encuestas (Anexo 1) con 13 preguntas cerradas acerca del uso del ABP por parte de los estudiantes del grupo 1 y 2 de la carrera de Psicología de sexto nivel que cursan la materia de trastornos de personalidad. Según Ismaru (2015), las preguntas cerradas en una encuesta son aquellas preguntas en donde el encuestado tiene que elegir entre las opciones establecidas y seleccionar una sola respuesta acorde a la actuación del sujeto. 


\section{RESULTADOS}

Una vez aplicada la encuesta a la muestra establecida podemos concluir varios puntos. Para empezar, de los 76 estudiantes encuestados, el 61,8 \% indica que conocen lo que es el aprendizaje basado en problemas, lo que significa que los estudiantes saben acerca de esta herramienta que utilizaron durante este período académico, pues fueron los protagonistas de su propio aprendizaje durante cuatro meses. Para ello se siguió un proceso y se cumplieron ciertos parámetros.

Como inicio, el 77,6\% de los encuestados aseguran que los objetivos de aprendizaje para cada actividad y tema a tratar, semana a semana, fueron claramente definidos por el maestro encargado de la materia, por lo cual, se logró una mejor comprensión de la materia y su relación con el ámbito profesional, siendo este un porcentaje de $84,2 \%$ según los estudiantes. Cabe destacar que, para el cumplimiento de este parámetro, los estudiantes tenían al alcance tanto el uso de redes sociales como del acompañamiento del tutor.

Por otro lado, al ser este tipo de aprendizaje, un potenciador para mejorar las relaciones interpersonales, pudimos constatar que, en efecto, mediante el uso del ABP, el $75 \%$ de los estudiantes encuestados aumentaron sus ha- bilidades sociales y comunicativas con sus compañeros y su tutor de clase, así como también, consiguieron una considerable mejora en el trabajo en equipo para cumplir semana a semana con las tareas y actividades establecidas.

Para continuar, se puede constatar el cumplimiento de los objetivos del ABP al conocer que, según el $77,6 \%$ de encuestados, el docente planteó preguntas que estimularon el pensamiento y análisis sobre la información revisada en clase. Y por ello las actividades prácticas fueron un buen complemento a los contenidos teóricos de la materia según el $88,2 \%$ de estudiantes encuestados. Para terminar, se señala que también se les permitió a los estudiantes evaluar su propio desempeño según los criterios establecidos por el docente.

Sin embargo, este método no estuvo exento de problemas, pues cada herramienta académica puede llegar a presentar algunas dificultades, tal es el caso del $28,8 \%$ de estudiantes que señalaron presentar dificultades en el ámbito de trabajo en equipo o el hecho de disponer de tiempo insuficiente para realizar las tareas establecidas, mas, cabe destacar que este es un porcentaje mínimo el cual no incide en la efectividad del uso del ABP, ya que el $71,1 \%$ de encuestados afirma la importancia de uso del méto- 
do para comprender y crear interés en la asignatura y los temas desarrollados.

Finalmente, el 89,5\% de estudiantes afirma que el uso del ABP ha contribuido a que la materia se les haga interesante y les ha permitido aprender aspectos que les ayudarán a desenvolverse en la vida laboral en un futuro, como por ejemplo: el trabajo en equipo, mejorar las relaciones interpersonales, la capacidad para solucionar los problemas y cumplir con las tareas y roles asignados en un determinado entorno. Por el hecho de que, para el $75 \%$ de encues- tados, los contenidos vistos en clase fueron apropiados para el nivel educativo que cursan, relacionado al cumplimiento de objetivos del ABP, es que el $64,5 \%$ de encuestados indica sentirse deseosos de implementar la metodología del ABP en otras asignaturas, a futuro.

Con este análisis podemos observar resultados muy positivos en su mayoría que atribuyen que el uso del ABP es una herramienta académica útil y necesaria para los estudiantes de sexto nivel de la carrera de Psicología que cursan la materia de trastornos de personalidad.

\section{CONCLUSIONES}

Al utilizar el método ABP en las instituciones educativas a nivel superior, se puede observar que este tipo de técnica de enseñanza ayuda al estudiante a plantearse mejores objetivos, a desarroIlar abiertamente sus habilidades, permitiendo que los estudiantes desarrollen capacidades y aptitudes como la comunicación, la responsabilidad, la toma de decisiones no solo en el ámbito escolar, sino también en el ámbito personal y profesional.

El uso de esta técnica ha dado como resultados promover el interés y la indagación a los y las estudiantes a investigar ciertos temas para un aprendizaje innovador, didáctico, con el fin de adquirir un mejor conocimiento autónomo.

Por lo tanto el papel del estudiante es autónomo en esta nueva metodología, sin embargo, el papel del tutor es fundamental, según el estudio aplicado a los y las estudiantes de psicología de sexto nivel manifestaron que las tutorías en las horas de clase, semana a semana, en cada actividad fue de gran ayuda para entender y aplicar las técnicas y estrategias que requerían este método.

Según las respuestas de la metodología aplicada se evidencia el impacto y las oportunidades que el método Aprendizaje Basado en Problemas trajo a los y las estudiantes, dejando de lado 
el método tradicional, los y las estudiantes aprendieron o reforzaron habilidades y aptitudes; como relaciones interpersonales, trabajo en equipo, habilidades sociales y comunicativas con sus compañeros, lo cual tendrá como objetivo potenciar dichos conocimientos en el campo profesional.

Cabe mencionar que este nuevo método de aprendizaje puso a prueba a los y las estudiantes universitarios, poniendo a prueba sus capacidades y conocimientos a nivel individual y grupal, es por ello que, según el estudio, la mayor parte de los estudiantes desearían ejecutar este nuevo método en otras materias.

El trabajo realizado nos ha permitido dar a conocer y comprobar el impacto de una nueva metodología impartida por los y las docentes a nivel educativo, la cual nos ha permitido potenciar conocimientos como estudiantes de tercer nivel. 


\section{REFERENCIAS}

Carretero, M. (1993). Constructivismo y educación. Madrid: Edelvives.

Chavez, G., Gonzalez, B., \& Hidalgo, C. (2016). Aprendizaje Basado en Problemas (ABP) a través del m-learning para el abordaje de casos clínicos. Innovación Educativa, 95-112.

Fernandez, L. (2016). Aprendizaje Basado en Problemas. Medisan .

Ismaru. (2015). Obtenido de https:// www.e-encuesta.com/tipos-de-pregunta-en-la-encuesta/ Morales, P. (2018). aprendizaje basado en problemas. Revista Electrónica Interuniversitaria de profesorado, 91-108.

Restrepo, B. (2005). Aprendizaje basado en problemas. pedagogía universitaria, 9-19.

Sanz, R. (2017). Obtenido de https://cursos.com/metodo-cuantitativo/ 


\section{ANEXO 1}

\section{TABULACIÓN DE RESULTADOS}

\section{Gráfico 1}

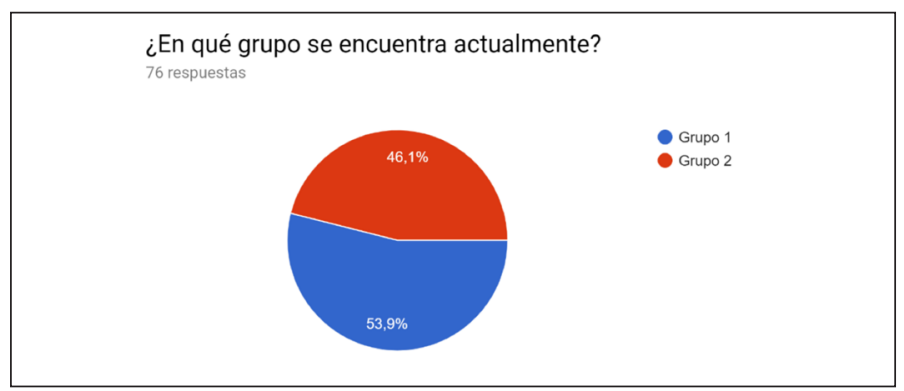

Elaborado por Paula Lárraga, Jessy Robayo, Belén Miño, Alejandra Díaz

- $\quad$ El 46,1\% de encuestados se encuentran en el grupo 2

- $\quad$ El 53,9\% de encuestados se encuentran en el grupo 1

\section{Gráfico 2}

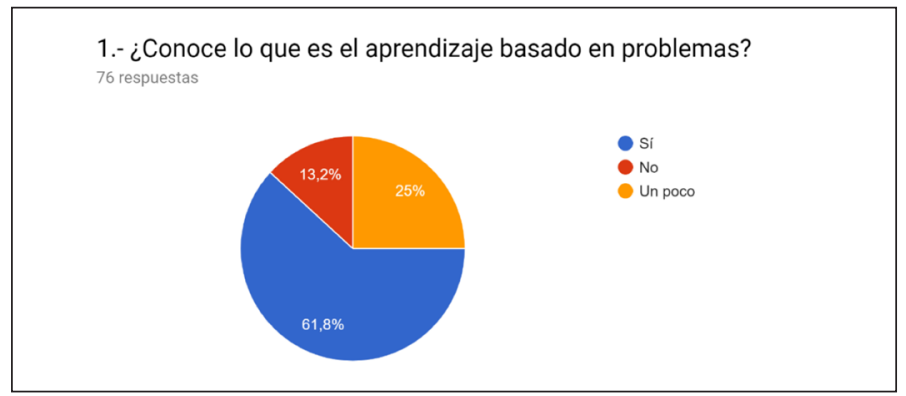

Elaborado por Paula Lárraga, Jessy Robayo, Belén Miño, Alejandra Díaz

- $\quad$ El 61,8\% de encuestados conocen el aprendizaje basado en problemas

- $\quad$ El $25 \%$ de encuestados conocen un poco sobre el aprendizaje basado en problemas

- $\quad$ El 13,2\% de encuestados no conocen el aprendizaje basado en problemas 


\section{Gráfico 3}

2.- ¿Los contenidos vistos fueron apropiados para el nivel educativo de los estudiantes?

76 respuestas
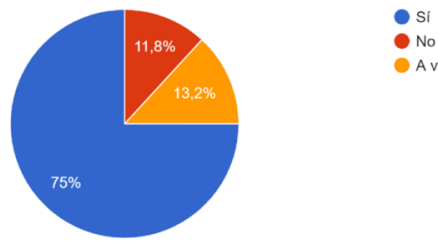

No

A veces

Elaborado por Paula Lárraga, Jessy Robayo, Belén Miño, Alejandra Díaz

- $\quad$ El $75 \%$ de encuestados aseguran que los contenidos vistos fueron apropiados para el nivel educativo de los estudiantes

- $\quad$ El 13,2\% de encuestados aseguran que los contenidos vistos fueron apropiados para el nivel educativo de los estudiantes, a veces

- $\quad$ El 11,8\% de encuestados aseguran que los contenidos vistos no fueron apropiados para el nivel educativo de los estudiantes

\section{Gráfico 4}

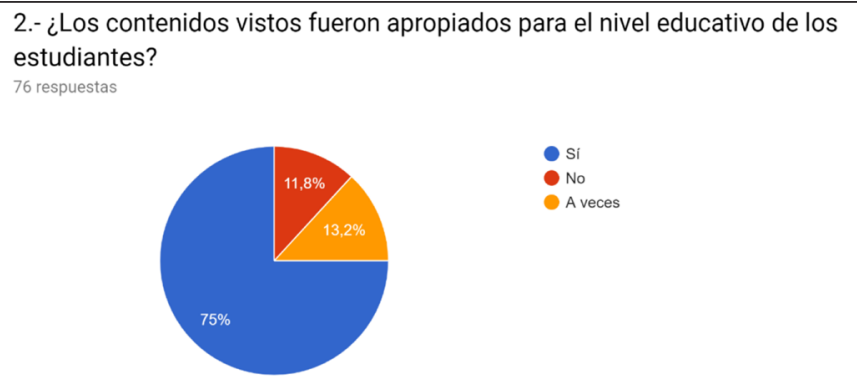

Elaborado por Paula Lárraga, Jessy Robayo, Belén Miño, Alejandra Díaz

- $\quad$ El 77,6\% de encuestados asegura que los objetivos de aprendizaje para cada actividad fueron claramente definidos

- El 13,2\% de encuestados asegura que los objetivos de aprendizaje para cada actividad fueron claramente definidos, a veces 
- $\quad$ El 9,2\% de encuestados asegura que los objetivos de aprendizaje para cada actividad no fueron claramente definidos

\section{Gráfico 5}

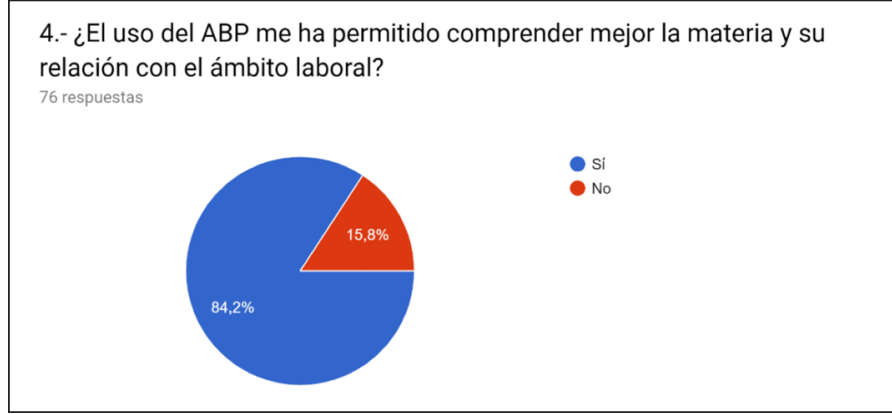

Elaborado por Paula Lárraga, Jessy Robayo, Belén Miño, Alejandra Díaz

- $\quad$ El 84,2 \% de encuestados aseguran que el uso del ABP les ha permitido comprender mejor la materia y su relación con el ámbito laboral

- $\quad$ El 15,8\% de encuestados aseguran que el uso del ABP no les ha permitido comprender mejor la materia y su relación con el ámbito laboral

\section{Gráfico 6}
5.- ¿Cree usted que el ABP aumenta las habilidades sociales y comunicativas de los estudiantes?
76 respuestas

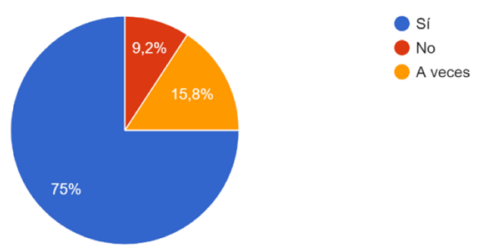

Elaborado por Paula Lárraga, Jessy Robayo, Belén Miño, Alejandra Díaz

- $\quad$ El $75 \%$ de encuestados creen que el ABP aumenta las habilidades sociales y comunicativas de los estudiantes

- $\quad$ El 15,8\% de encuestados creen que el ABP no aumenta las habilidades sociales y comu- 
nicativas de los estudiantes

- $\quad$ El 9,2\% de encuestados creen que el ABP aumenta las habilidades sociales y comunicativas de los estudiantes, a veces

\section{Gráfico 7}

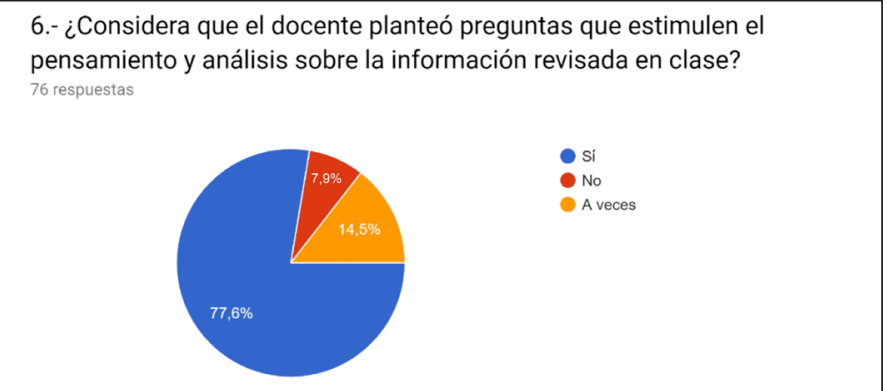

Elaborado por Paula Lárraga, Jessy Robayo, Belén Miño, Alejandra Díaz

- $\quad$ El 77,6 \% de encuestados consideran que el docente planteó preguntas que estimulen el pensamiento y análisis sobre la información revisada en clase

- $\quad$ El 14,5 \% de encuestados consideran que el docente planteó, a veces, preguntas que estimulen el pensamiento y análisis sobre la información revisada en clase

- $\quad$ El 7,9\% de encuestados consideran que el docente no planteó preguntas que estimulen el pensamiento y análisis sobre la información revisada en clase

\section{Gráfico 8}

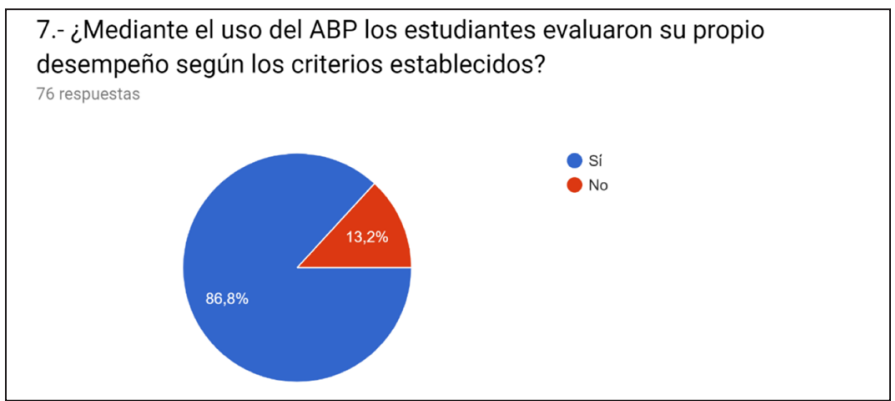

Elaborado por Paula Lárraga, Jessy Robayo, Belén Miño, Alejandra Díaz 
- El $86,8 \%$ de estudiantes aseguran que mediante el uso del ABP los estudiantes evaluaron su propio desempeño según los criterios establecidos

- El 13,2\% de estudiantes aseguran que mediante el uso del ABP los estudiantes no evaluaron su propio desempeño según los criterios establecidos

\section{Gráfico 9}

8.- ¿Las actividades prácticas fueron un buen complemento a los contenidos teóricos de la materia?

76 respuestas

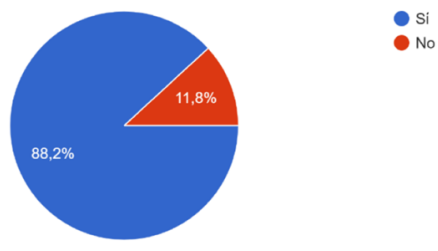

Elaborado por Paula Lárraga, Jessy Robayo, Belén Miño, Alejandra Díaz

- El 88,2\% de estudiantes aseguran que las actividades prácticas fueron un buen complemento a los contenidos teóricos de la materia

- El 11,8\% de estudiantes aseguran que las actividades prácticas no fueron un buen complemento a los contenidos teóricos de la materia

\section{Gráfico 10}

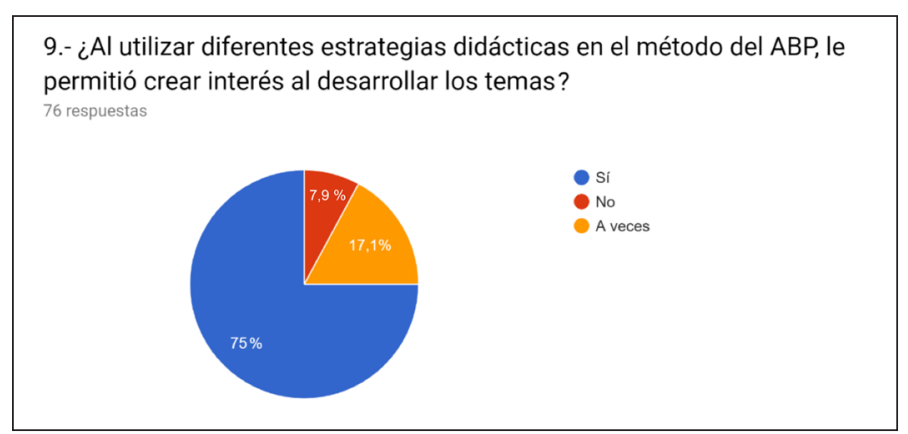

Elaborado por Paula Lárraga, Jessy Robayo, Belén Miño, Alejandra Díaz

- El $75 \%$ de encuestados creen que al utilizar las diferentes estrategias didácticas en el 
método del ABP, les permitió crear interés al desarrollar los temas

- $\quad$ El 17,1\% de encuestados creen que al utilizar las diferentes estrategias didácticas en el método del ABP, no les permitió crear interés al desarrollar los temas

- $\quad$ El 7,9\% de encuestados creen que al utilizar las diferentes estrategias didácticas en el método del ABP, les permitió crear interés al desarrollar los temas, a veces

\section{Gráfico 11}

10.- ¿Presentó alguna de las siguientes dificultades en el transcurso del semestre con el uso del ABP?

76 respuestas

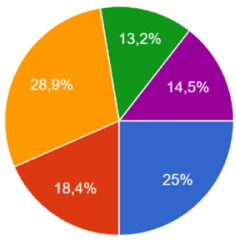

Confusión sobre las tareas

Poco tiempo para realizar las actividades

Mal trabajo en equipo

Instrucciones deficientes por parte del

tuto

Ninguna

Elaborado por Paula Lárraga, Jessy Robayo, Belén Miño, Alejandra Díaz

- $\quad$ El 28,9\% de encuestados aseguran haber tenido dificultades en el trabajo en equipo

- $\quad$ El $25 \%$ de encuestados aseguran haber tenido confusión sobre las tareas

- $\quad$ El 18,4 \% de encuestados aseguran haber tenido dificultades al tener poco tiempo para realizar las actividades

- $\quad$ El 14,5\% de encuestados aseguran no haber tenido dificultad alguna

- El 13,2\% de encuestados aseguran haber recibido instrucciones deficientes por parte del tutor 


\section{Gráfico 12}

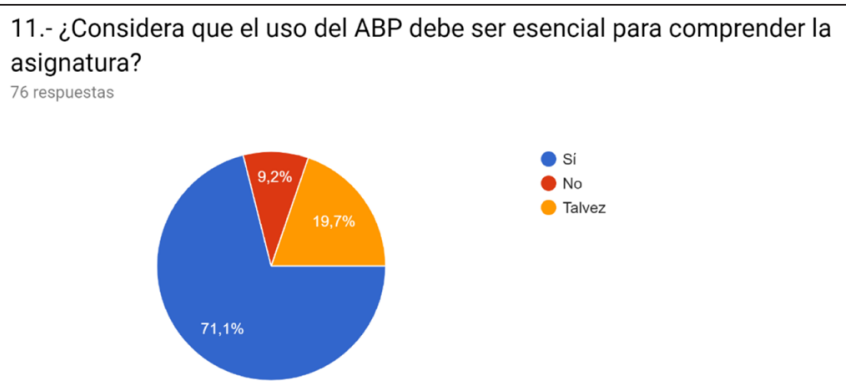

Elaborado por Paula Lárraga, Jessy Robayo, Belén Miño, Alejandra Díaz

- $\quad$ El 71,1\% de encuestados consideran que el uso del ABP debe ser esencial para comprender la asignatura

- $\quad$ El 19,7 \% de encuestados consideran que talvez el uso del ABP debe ser esencial para comprender la asignatura

- $\quad$ El 9,2 \% de encuestados no consideran que el uso del ABP debe ser esencial para comprender la asignatura

\section{Gráfico 13}

12.- El uso del ABP en la materia me ha parecido interesante y me ha permitido aprender aspectos para desempeñarme en mi vida laboral en un futuro?

76 respuestas

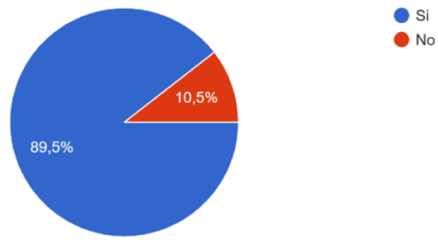

Elaborado por Paula Lárraga, Jessy Robayo, Belén Miño, Alejandra Díaz

- $\quad$ El 89,5 \% de encuestados aseguran que el uso del ABP en la materia ha sido interesante y les ha permitido aprender aspectos para desenvolverse en la vida laboral en el futuro

- El 10,5\% de encuestados aseguran que el uso del ABP en la materia no ha sido interesante y no les ha permitido aprender aspectos para desenvolverse en la vida laboral en el futuro 


\section{Gráfico 14}

13.- ¿Desearía implementar la metodología del ABP en otras asignaturas? 76 respuestas
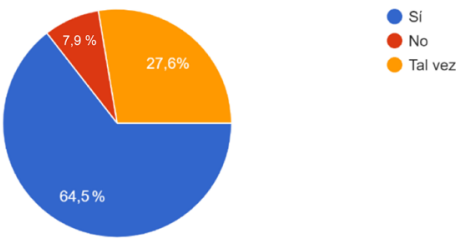

Elaborado por Paula Lárraga, Jessy Robayo, Belén Miño, Alejandra Díaz

- $\quad$ El 64,5 \% de encuestados desearían implementar el uso del ABP en otras asignaturas

- $\quad$ El 27,6 \% de encuestados tal vez desearían implementar el uso del ABP en otras asignaturas

- $\quad$ El 7,9\% de encuestados no desearían implementar el uso del ABP en otras asignaturas 